\title{
Polarization imaging over sea surface - a method for measurements of Stokes components angular distribution
}

W. Freda

wfreda@am.gdynia.pl

\section{J. Piskozub}

\section{H. Toczek}

Gdynia Maritime University, ul. Morska 81-87, 81-225 Gdynia, Poland

Institute of Oceanology PAS, ul. Powstańców Warszawy 55, 81-712 Sopot, Poland

This article describes a method for determining the angular distribution of light polarization over a roughened surface of the sea. Our method relies on measurements of the Stokes vector elements using a polarization imaging camera that operates using the Division of Focal Plane (DoFP) method. It uses special monochrome CCD array in which the neighbouring cells, instead of recording different colours (red green and blue), are equipped with micropolarizers of four directions (0,45, 90 and 135 degrees).

We combined the camera with a fish-eye lens of Field of View (FoV) $>180$ deg. Such a large FoV allowed us to crop out the fragment of the frame along the circular horizon, showing a view covering all directions of the hemisphere. Because of complicated optical design of the fish-eye lens (light refraction on surfaces of parts of the lens) connected to the sensor we checked the accuracy of the measurement system. A method to determine the accuracy of measured polarization is based on comparison of the experimentally obtained rotation matrix with its theoretical form. Such a comparison showed that the maximum error of Stokes vector elements depended on zenith angle and reached as much as $24 \%$ for light coming from just above the horizon, but decreased rapidly with decreasing zenith angle to the value of $12 \%$ for the angles $10^{\circ}$ off the edge of FoV.

Moreover we present the preliminary results prepared over rough sea surface. These results include total intensity of light, Degree of Linear Polarization (DoLP) and their standard deviations. The results have been averaged over one thousand frames of a movie. These results indicate that the maximum polarization is observed near the reflection of the sun, and the signal coming from below the surface may be observed at zenith angles far from the vertical direction.

[DOI: http://dx.doi.org/10.2971/jeos.2015.15060]

Keywords: Polarization imaging, upwelling radiance, degree of linear polarization, sea surface

\section{INTRODUCTION}

Polarization properties of light in atmosphere-ocean system were an object of interest since the 1960s. The early studies, based mainly on Monte Carlo modelling, presented results about the degree of polarization, variability and an angle of polarization for light reflected and transmitted through cloud or haze layers (see Deirmendjian [1], Kattawar and Plass [2, 3]). They were followed by the first papers describing radiance and its degree of polarization in complex atmosphere-ocean system at the Top Of Atmosphere (TOA), as well as just above and below ocean surface (Kattawar [4]). In the next decade, first studies about complete Stokes vector for atmosphere-ocean system have been published e.g. Kattawar and Adams [5].

After the 1980s there was a decline in interest in this topic resulting in lower number of papers. However today, when technical capabilities have been increased significantly, polarization is again in the spotlight. Polarization properties of light seems to carry useful information, especially for ocean colour remote sensing. Previous studies, based mainly on radiative transfer modelling, have shown that polarisation properties of radiation measured above water surface is sensitive to concentration of suspended particular matter (Chami et al. [6], Chami and McKee [7]). Moreover, polarization may be used to separate the inorganic and biogenic parts of suspended matter (see Gilerson et al. [8], Chami [9] and Tonizzo et al. [10]). It has been shown by Piskozub and Freda [11], that degree of polarization over sea surface strongly depends on single scattering albedo changes caused by various concentrations of bubbles. It is also well known that reflected light is partly polarized, that is why polarization can significantly improve the quality of signal coming from below surface when measured above the seawater. Hence the concept of He et al. [12] of parallel polarization radiance (PPR) acquisition, that reduces the sun glint in received signal and increases the ocean colour signal at the top of atmosphere for some viewing directions.

Almost all above mentioned studies were based on radiative transfer simulations. That is why we are particularly interested in the experimental confirmation of polarization phe- 
nomena which have been predicted by modelling. In this paper, we present our first results of measurements of the Stokes vector components angular distribution, performed over the sea surface. Moreover a substantial part of the article involves a determination of the measurement accuracy that takes into account the impact of polarization properties of the fish-eye lens connected with a polarization receiver.

\section{METHODS}

Until recently the imaging of polarization properties of light reflected from waving ocean surface or fast moving objects was difficult, in particular, when the information was needed about three components of the Stokes vector (linear polarization) or all four components (complete description of both linear and circular polarization). Polarization detectors used earlier were based on rotating polarizing elements (as quarterwave plates) or photoelastic elements [13] that made it possible to measure the elements of Stokes vector in successive time intervals only. This approach was useful for scattering matrix measurements (see for example Voss and Fry [14] or Hielscher et al. [15]), but turned out to be useless in imaging of dynamically changing sea surface.

Recently other methods for simultaneous imaging of elements of the Stokes vector have been developed. A review of such methods was given by Tyo et al. [16].

Similar application of polarization imaging but for underwater downwelling radiance were studied by Voss and Souaidia [17] and Bhandari [18]. Their methods rely on using three separate CCD matrices with three fish-eye lenses [17] or one CCD matrix receiving the light coming from four separate lenses via special optical fibres [18].

The method we chose was Division of Focal Plane (DoFP). It used special monochrome CCD array in which the neighbouring cells, instead of recording different colours (like in ordinary camera), were equipped with micropolarizers of four directions. Our sensor was PolarCam ${ }^{\mathrm{TM}}$ with $1208 \times 1400$ of usable pixels produced by 4D Technology (www.4dtechnology.com) and its main advantages were small size, possibility of portable battery power and full control with a laptop type computer. The same type of polarization imaging camera has been applied to check a capability to distinguish the oil from the water during a surface spill [19].

The micropolarizer array, described by Brock et al. [20], contained a pattern of polarizers with four directions $(0,45,90$ and 135 degrees). Single pixel is $7.4 \times 7.4 \mu \mathrm{m}$ size, and the micropolarizers matched to detector pixels are made with nanoscale patterning. They formed a metal grating with subwavelength spacing placed on a thin transparent glass. The Stokes vector components were obtained as follows:

$$
\mathbf{S}=\left[\begin{array}{c}
I \\
Q \\
U \\
V
\end{array}\right]=\left[\begin{array}{c}
I_{0}+I_{90} \\
I_{0}-I_{90} \\
I_{45}-I_{135} \\
I_{L H C}-I_{R H C}
\end{array}\right]
$$

where $I_{0}, I_{45}, I_{90}, I_{135}$ are the intensities of the linear polarization components of $0,45,90$ and 135 degrees, while $I_{L H C}$ and
$I_{R H C}$ are the intensities of left hand and right hand circular polarization components. The sensor was designed to determine three components of Stokes vector, that is $I, Q$ and $U$. Additional waveplate would have allowed to obtain circular polarization, but such a choice would have complicated the acquisition and excluded the simultaneity of measurement. Fortunately, circular polarization effects are really small in seawater (see off-diagonal elements of Mueller matrices [14]) and hence can be neglected. The collected images make it possible to obtain the Degree of Linear Polarization (DoLP):

$$
\operatorname{DoLP}=\frac{\sqrt{Q^{2}+U^{2}}}{I}
$$

Components $I$ and $V$ of Stokes vector are independent of coordinate system, but $\mathrm{Q}$ and $\mathrm{U}$ depend on the orientation of observation plane. For a light beam described by the Stokes vector $\mathbf{S}=\left[\begin{array}{llll}I & Q & U & V\end{array}\right]^{\mathrm{T}}$ (superscript $\mathrm{T}$ denotes transposition) for a certain orientation, the Stokes vector $\mathbf{S}^{\prime}$ after rotation through an angle $\phi$, that can be described as $\mathbf{S}^{\prime}=\mathbf{R}(\phi) \mathbf{S}$, takes the form of:

$$
\left[\begin{array}{c}
I^{\prime}(\phi) \\
Q^{\prime}(\phi) \\
U^{\prime}(\phi) \\
V^{\prime}(\phi)
\end{array}\right]=\left[\begin{array}{cccc}
1 & 0 & 0 & 0 \\
0 & \cos (2 \phi) & -\sin (2 \phi) & 0 \\
0 & \sin (2 \phi) & \cos (2 \phi) & 0 \\
0 & 0 & 0 & 1
\end{array}\right]\left[\begin{array}{c}
I \\
Q \\
U \\
V
\end{array}\right]
$$

Such a rotation matrix $\mathbf{R}(\phi)$ was used for determination of a quality of received Stokes parameters and in the following section is compared with the experimentally received form.

We equipped the polarization imaging camera with the fisheye lens Fujinon FE 185C086HA-1. Such a lens produced a round view of FoV of 185 degrees, that has the shape of a circle of $8.6 \mathrm{~mm}$ diameter on the CCD matrix. Such a view was cropped along the horizon line to receive an image coming from a whole hemisphere. Images and movies were recorded with PolarView software, and then they were processed in Matlab Image Processing Toolbox to determine zenith and azimuth coordinates. All results were presented as polar graphs and their angular resolution reduced to 1 degree (both zenith and azimuth). The results obtained from the measurements of the polarization imaging camera were supplemented by parameters of wind speed and wind direction obtained from the on-line data available at [21] for the vicinity of the measurement station. Moreover the angular position of the sun (zenith and azimuth) was obtained from Solar Position Calculator provided by NOAA ESRL [22].

\section{RESULTS}

The results presented here are divided into the following subsections: the verification of the linear mapping of zenith angles on the CCD matrix (Subsection 3.1), the analysis of polarization uncertainty for the view of the hemisphere and the resulting errors introduced by measurement system (Subsection 3.2). Sequent subsection includes the results of measurements of DoLP of sky and averaged results of upwelling light recorded over a roughened surface of the sea. It contain total intensity, DoLP and their standard deviations. 


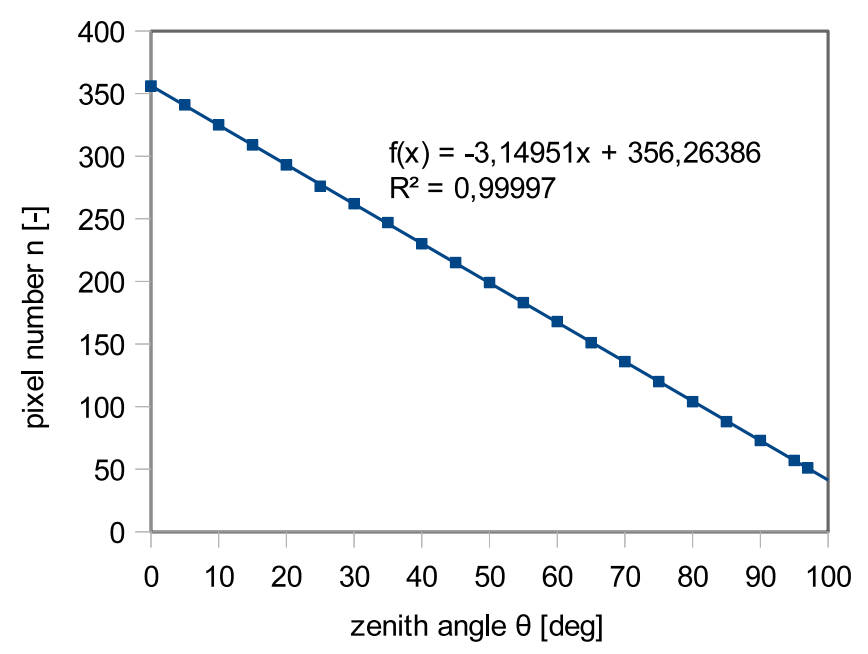

FIG. 1 Spatial distribution (expressed by pixel numbers) of light beam, that reaches the CCD matrix of PolarCamTM equipped with the fish-eye lens from various zenith directions.

\subsection{View of the hemisphere}

Because of complicated optical design of the fish-eye lens (light refraction on surfaces of parts of lens) we have checked the geometrical quality of lens to examine its image deformation. The camera is placed on the precise rotation mount in front of the point source of light and during rotation (zenith angle $\theta$ change) the respective numbers of the lightened pixels are recorded (Figure 1). Each of the pixels presents one (of four) polarization mode only - therefore the whole CCD matrix of size $1208 \times 1400$ pixels has been divided into four views of $604 \times 700$ pixels.

Such a calibration confirmed the precise linear distribution on the CCD matrix, and additionally gave information about the size of the Field of View (FoV) on the CCD matrix. It was derived, from the slope of linear relation shown in the Figure 1, that the fish-eye lens produces a $180^{\circ} \mathrm{FoV}$ on the circle of 567 pixels diameter (measured after division of the matrix into four polarizations). That produced the angular resolution of about $0.3^{\circ}$ which, when averaged in Matlab, was reduced to 1 degree.

\subsection{Impact of the polarization imaging system}

Another important step was to check the influence of the imaging system on measured polarization. This influence may be due to limited quality of micropolarizers attached to the sensor as well as refraction on the surfaces of parts of the fisheye lens.

Despite the axial symmetry of the lens, its polarization influence can not be limited to establish the relation of the camera rotation around vertical axis (angle $\theta$ ). It turned out that rotation of the camera around optical axis of the mounted fisheye lens caused different responses from variability of Stokes vector elements. Because of that, we have installed our polarization imaging camera on precise rotating mount in front of unpolarized light source covered with wire grid polarizer (Thorlabs) see Figure 2. That gave us the possibility to change

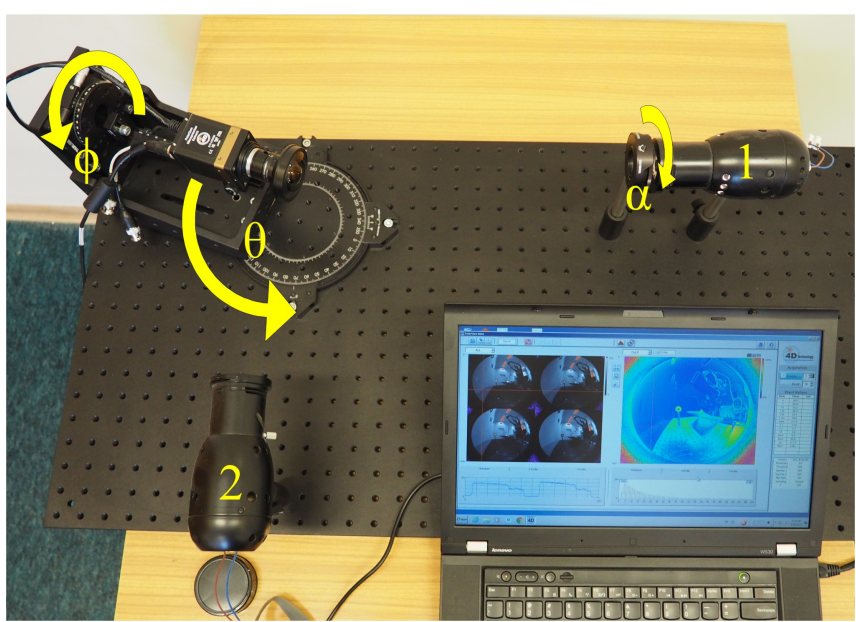

FIG. 2 The calibration system: polarization imaging camera is placed on a precise rotation mount, that allow to change both angles of view $\theta$ and $\phi$. Source of light 1 is covered with polarizer, that polarization plane $\alpha$ may be adjusted. The second source of light 2 is mounted for the precise location of the camera. Additionally, the computer with PolarView ${ }^{\mathrm{TM}}$ software is visible.

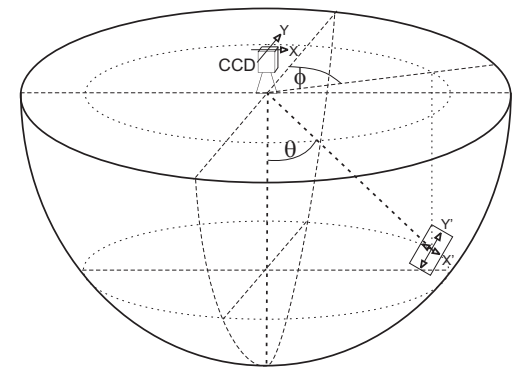

FIG. 3 The coordinate system for the view from a camera equipped with the fish-eye lens during field measurements.

zenith angle of the light source $\theta$. Additionally, the second rotating mount allowed us to rotate the camera around the optical axis of the lens (changes of azimuth angle of view $\phi$ ) - see Figure 3.

Taking into account the impact of both the lens and the characteristic of microplarizers required to obtain the angular distribution of the experimental rotation matrix $\mathbf{C}$, which transforms the Stokes vector of light coming from the environment $\mathbf{S}_{\text {inc }}$ into the Stokes vector of light received at the CCD matrix $\mathbf{S}_{\text {rec }}$.

$$
\left[\begin{array}{l}
I(x, y) \\
Q(x, y) \\
U(x, y) \\
V(x, y)
\end{array}\right]_{r e c}=\mathbf{C}(\theta, \phi)\left[\begin{array}{c}
I(\theta, \phi) \\
Q(\theta, \phi) \\
U(\theta, \phi) \\
V(\theta, \phi)
\end{array}\right]_{i n c}
$$

where $(x, y)$ are coordinates of pixel recording light arriving from the direction given by angles $(\theta, \phi)$ that denote zenith and azimuth respectively.

The experimental rotation matrix matrix $\mathbf{C}$ can not be treated as a Mueller matrix of the fish-eye lens because beside information about polarization impact of the lens, it is influenced also by the CCD matrix characteristic and rotation of the $(X ; Y)$ coordinates of the CCD matrix into local coordinates $\left(X^{\prime} ; Y^{\prime}\right)$ that depend on a view direction $(\theta, \phi)$. 
Calculation of the experimental rotation matrix matrix elements required us to perform the following consideration. Signals were recorded for four directions of polarizer and for various angular direction of the camera. Such a situation allow us to describe it by equation

$$
\left[\begin{array}{c}
I(x, y) \\
Q(x, y) \\
U(x, y) \\
V(x, y)
\end{array}\right]_{r e c}=\mathbf{C}(\theta, \phi) \mathbf{M}_{p}\left[\begin{array}{c}
I(\theta, \phi) \\
0 \\
0 \\
0
\end{array}\right]_{i n c}
$$

where $\mathbf{M}_{p}$ is a Mueller matrix of polarizer. Polarization directions of our camera are expressed in relation to the vertical axis (clockwise), direction consistent with the $\mathrm{Y}$ axis means polarization angle $\alpha=0$. Hence putting an ideal linear polarizer in front of the camera flashed by unpolarized light should result in Stokes vectors of light coming toward the lens:

- vertical direction of polarization plane $\left(\alpha=0^{\circ}\right)$ :

$$
\mathbf{M}_{p} I_{\text {inc }}(\mid)=I_{\text {inc }}\left[\begin{array}{llll}
1 & -1 & 0 & 0
\end{array}\right]^{T}
$$

- horizontal direction of polarization plane $\left(\alpha=90^{\circ}\right)$ :

$$
\mathbf{M}_{p} I_{\text {inc }}(-)=I_{\text {inc }}\left[\begin{array}{llll}
1 & 1 & 0 & 0
\end{array}\right]^{T}
$$

- oblique direction of polarization plane $\left(\alpha=45^{\circ}\right)$ :

$$
\mathbf{M}_{p} I_{\text {inc }}(/)=I_{\text {inc }}\left[\begin{array}{llll}
1 & 0 & 1 & 0
\end{array}\right]^{T}
$$

- oblique direction of polarization plane $\left(\alpha=135^{\circ}\right)$ :

$$
\mathbf{M}_{p} I_{\text {inc }}(\backslash)=I_{i n c}\left[\begin{array}{llll}
1 & 0 & -1 & 0
\end{array}\right]^{T}
$$

where lines in brackets depicts the direction of polarizer.

These vectors were applied to obtain the form of the experimental rotation matrix $\mathbf{C}$ :

$\mathbf{C}=\frac{I}{2 I_{\text {inc }}}\left[\begin{array}{cccc}I_{(\mid)}+I_{(-)} & I_{(\mid)}-I_{(-)} & I_{(/)}-I_{(\backslash)} & 0 \\ Q_{(\mid)}+Q_{(-)} & Q_{(\mid)}-Q_{(-)} & Q_{(/)}-Q_{(\backslash)} & 0 \\ U_{(\mid)}+U_{(-)} & U_{(\mid)}-U_{(-)} & U_{(/)}-U_{(\backslash)} & 0 \\ 0 & 0 & 0 & 1\end{array}\right]$

where the Stokes vector elements were calculated from formula (1) for four directions of polarizer and for various directions of light source $(\theta, \phi)$. The $I_{\text {inc }}$ value was obtained as the mean value of $I$, averaged for four different polarizations for each direction $(\theta, \phi)$ separately. Such a form of calibration matrix is not unique, and some of its elements (placed in the fist column) can be replaced by their different combinations. For example the $C_{11}=\left(I_{(\mid)}+I_{(-)}\right) /\left(2 I_{\text {inc }}\right)$ can be also obtained as $C_{11}=\left(I_{(/)}+I_{(\backslash)}\right) /\left(2 I_{i n c}\right)$. Such a consideration produces the experimental rotation matrix elements dependent on the zenith angle $\theta$, and azimuth angle $\phi$ (non-zero elements only). The raw data are shown in the Figure 4 for various azimuth angles $\phi$, and for one zenith angle $\theta$ equal to $70^{\circ}$.

The results of measurements were parametrized to obtain the calibration value for each of direction $(\theta, \phi)$, regardless of the angular resolution used during calculations. Results of parametrization is presented in the Figure 5. We are aware that this parametrization ignores errors associated with random differences (noise) of individual pixels, that is visible in the Figure 4.

The C matrix is influenced mainly by rotation (see Eq. (3)), however there are visible differences caused by both the direction dependent lens Mueller matrix and characteristics of

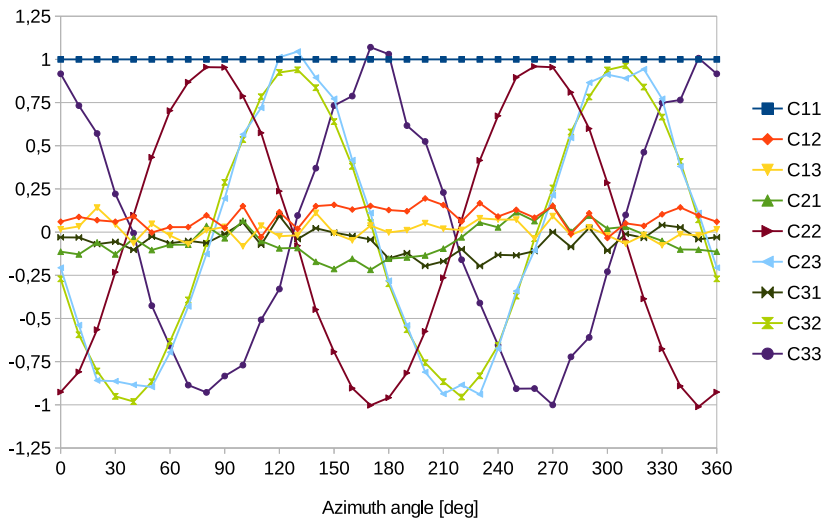

FIG. 4 Experimental rotation matrix elements plotted as a function of azimuth angles for zenith angle $\theta=70^{\circ}$.
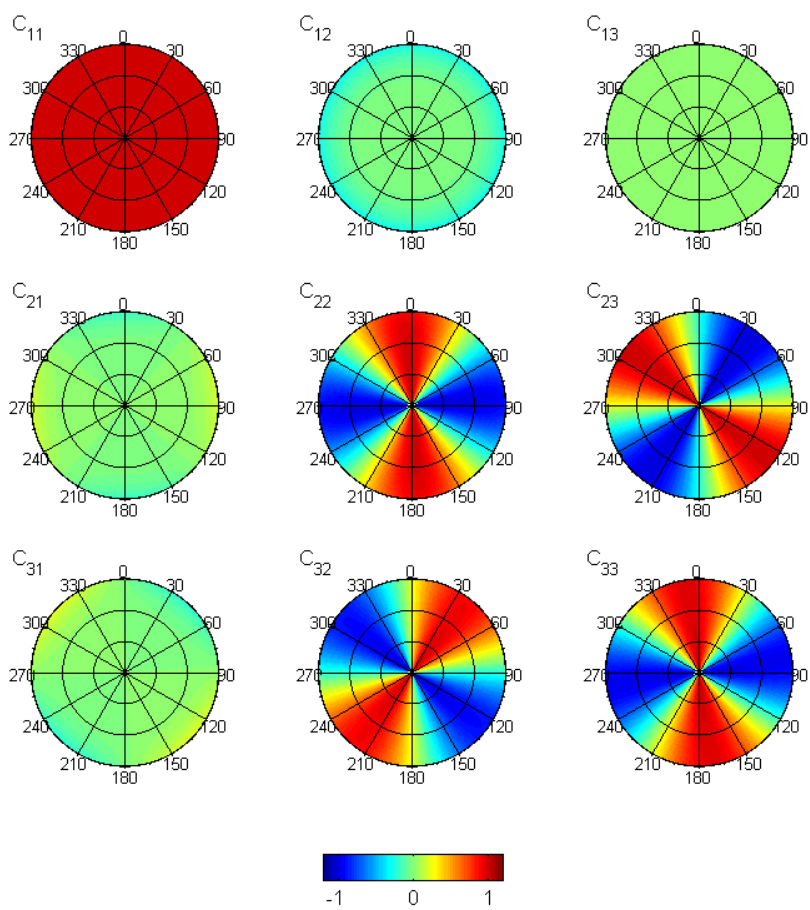

FIG. 5 Parametrization of experimental rotation matrix elements plotted as a function of azimuth and zenith angles of the hemisphere.

micropolarizers attached to the sensor. As a result of our calibration method the element $\mathbf{C}_{11}$ is equal to unity for all directions. The $\mathbf{C}_{13}$ element, which is shown as homologous to the theoretical rotation matrix (equal to 0 for all directions), originally slightly differed from zero. These small differences $(<0.1)$ showed a nature of noise which could not be described by any function of angle. Standard deviation of such a noise was about 0.06 , and probably depends on the parameters of lighting, aperture, shutter speed, etc. For that reason it was approximated to zero for all directions. The $\mathbf{C}_{12}$ element was independent of azimuth angles $\phi$, but varies from 0 in the middle of view $\left(\theta=0^{\circ}\right)$ to -0.32 at the edge of FoV.

We were surprised to find that the elements $\mathbf{C}_{21}$ and $\mathbf{C}_{31}$ are azimuth dependent, while adequate elements of the rotation matrix were simply zeros. The element $\mathbf{C}_{21}$, that is close to 0 in the middle of view, reaches a maximum of variability for the edge of FoV (zenith angle $\theta=90^{\circ}$ ), for which it varies from -0.23 (for azimuth $\phi$ close to $0^{\circ}$ and $180^{\circ}$ ) to 0.23 (for azimuth 
$90^{\circ}$ and $270^{\circ}$ ). The element $\mathbf{C}_{31}$, which is also azimuth dependent, reaches its maximum values of 0.21 for angles $\phi$ close to $130^{\circ}$ and $\phi=310^{\circ}$, and minimum values of -0.21 respectively for $\phi=40^{\circ}$ and $\phi=220^{\circ}$.

The variability of the other elements of calibration matrix $\mathbf{C}_{22}$, $\mathrm{C}_{23}, \mathrm{C}_{32}$ and $\mathrm{C}_{33}$ seems to be very similar to that of rotation matrix. However there are some differences, that can not be easily noticed in the Figure 3 . The element $\mathbf{C}_{22}$, that only slightly depend on zenith angle $\theta$, reaches the maximum value of 0.89 for the edge of FoV instead of 1 observed close to the centre of view for azimuth angles that are offset by $5^{\circ}$ in the counter-clockwise direction. Its maximum values stands for $\phi=355^{\circ}$ and $\phi=175^{\circ}$, while minimum values that reaches symmetrically from -0.89 (edge of field of view) to -1 in the centre, occurs for azimuth $\phi=85^{\circ}$ and $\phi=265^{\circ}$. Similar but even higher azimuth offset in the counter-clockwise direction is observed for elements $C_{23}$ and $C_{32}$ and $C_{33}$. It is respectively $8^{\circ}$ for both $\mathbf{C}_{23}$ and $\mathbf{C}_{32}$ and $11^{\circ}$ for $\mathbf{C}_{33}$ element. The $\mathbf{C}_{23}$ obtains higher range by $4 \%$ and has no zenith dependence. At the same time, the element $\mathbf{C}_{32}$ show a decrease in variation with zenith angle similar to $\mathrm{C}_{22}$.

The multiplication of the measured rotation matrix by the sample Stokes vectors resulted in the following results. Uncertainties of individual Stokes vector elements depend on the combination of $I, Q$ and $U$ values. The maximum uncertainties of elements of Stokes vector appeared on the edge of view and they reached even $24 \%$ for $\theta=90^{\circ}$. However, with the distance from the horizon these errors quickly decreased. They reached a maximum of $16 \%$ for an angle $\theta=85^{\circ}, 12 \%$ for $\theta=80^{\circ}$ and decreased below $6 \%$ for angles $\theta<70^{\circ}$. The origin of such discrepancies are refraction at parts of lens, that grows with zenith angle, polarization properties of anti reflection coating of parts of the lens and limited accuracy of micropolarizers.

\subsection{Results of preliminary measurements.}

Preliminary results of measurements of the Stokes vector elements distribution, performed over the sea surface are presented here. Figure 6 depicts the DoLP of sky recorded in cloudless weather. Direct light of sun, $22^{\circ}$ over the horizon, was obscured by a black cover (visible at the top of the view). Moreover one can recognize a lamp placed on the pier (on the left - around azimuth of $330^{\circ}$ ), and a mast (between 220 and 225 degrees). The view is dominated by the polarization pattern caused by molecular scattering (Rayleigh scattering), that reaches its maximum for directions perpendicular to the sun position $[23,24]$. Lower than theoretical values of DoLP are probably caused by atmospheric dusts and aerosols.

Comparison of this image with the expected results demonstrate that maximum errors resulting from the considerations made in Subsection 3.2 are not present in so large extent, even on the edge of view. The pattern of DoLP is mapped here with high accuracy, and the individual uncertainties of Stokes vector elements do not significantly affect the result of DoLP. A good mapping of DoLP is due to the fact that the main uncertainty is the effect of rotation only and it does not change the degree of linear polarization.

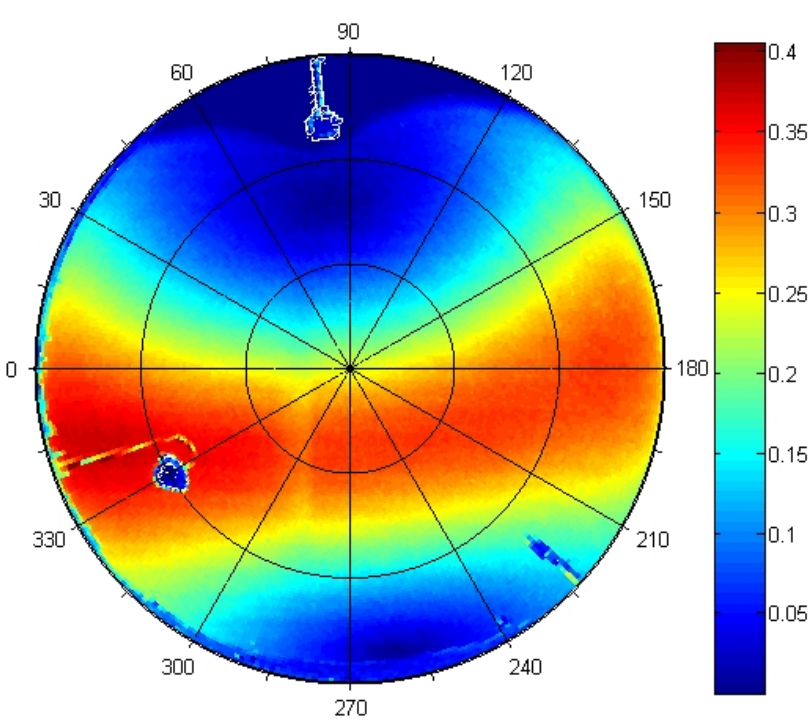

FIG. 6 DoLP of sky received for low sun position ( $22^{\circ}$ over horizon), no spectral filter is used.

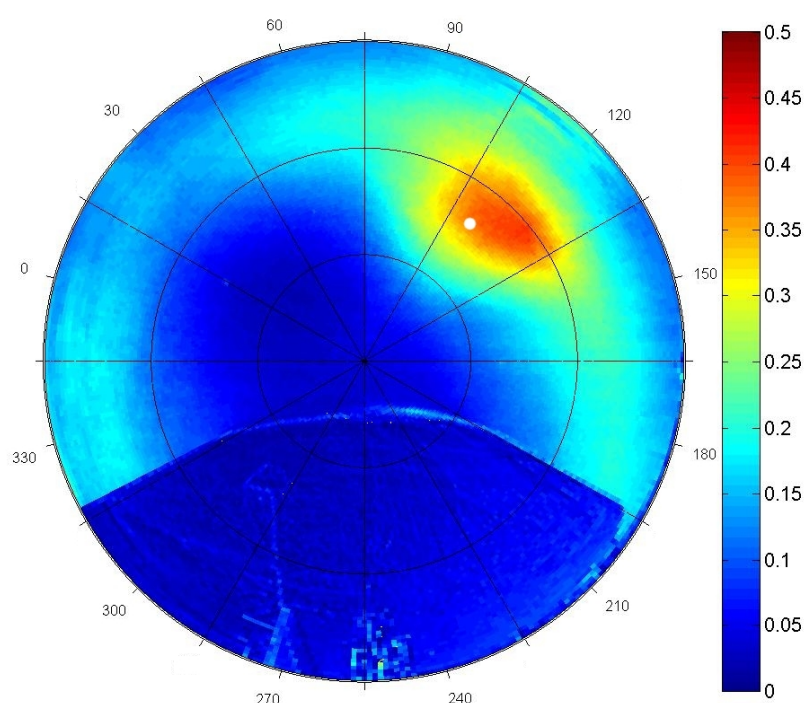

FIC. 7 DoLP of light received over sea surface for spectral filter $470 \mathrm{~nm}$. Exposure time $18 \mathrm{~ms}$. The figure presents average values of one thousand frames of the movie. Measured on 17 June 2015 at 10:34 (local time). Sun position $\theta=48^{\circ}, \phi=110^{\circ}$ (NOAA Solar Calculator). Wind speed was $6 \mathrm{~m} / \mathrm{s}$ (wind direction from $280^{\circ}$ relative to north).

Figure 7 depicts the DoLP of upwelling light over seawater. Measurements were made at the top of western breakwater in Łeba, Poland (54.77009N, 17.55089E). Despite using a camera crane (produced by Slide Kamera), one third of the view was obscured by a breakwater, on which the camera crane was set. Azimuth coordinates represent the compass scale ( 0 means north). The camera was directed $75^{\circ}$ to the east, so that the concrete construction of the breakwater occupied only the lower part of the picture. The position of direct sun reflection, which would be visible on the plain sea surface, has the coordinates $\theta=48^{\circ}, \phi=110^{\circ}$ (marked as white dot in the Figure 7). Wind of speed of $6 \mathrm{~m} / \mathrm{s}$ blew from the west direction $\left(280^{\circ}\right)$, which is typical in the southern Baltic region. Measurements were made with bandpass spectral filter mounted between the lens and the sensor. The maximum of transmittance was 470 nm, (MidOpt BP470) and the Full Width at Half Maximum (FWHM) was $85 \mathrm{~nm}$. 

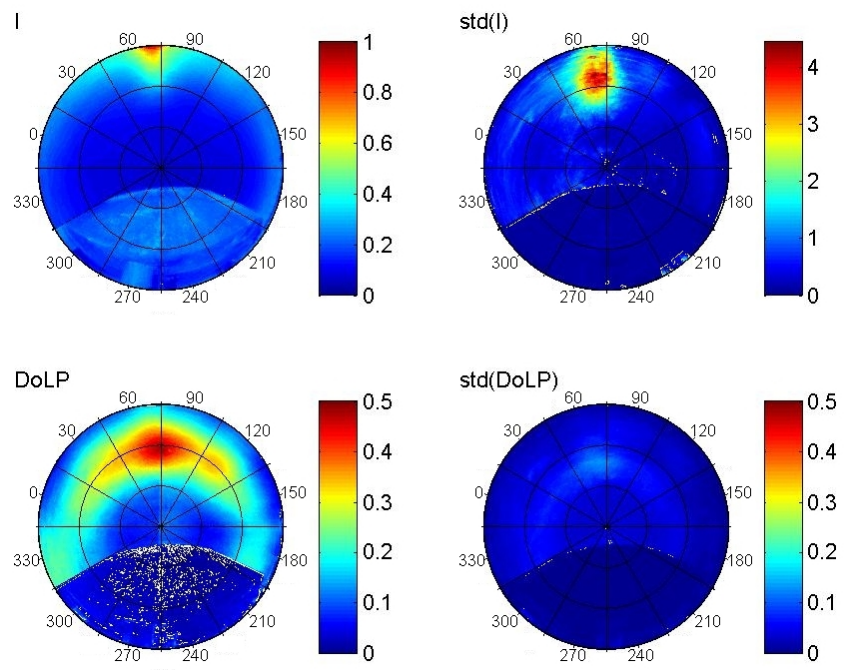

FIG. 8 Total intensity $I$, DoLP and their standard deviations of light received over sea surface for spectral filter $470 \mathrm{~nm}$. Exposure time $10 \mathrm{~ms}$. Average values of one thousand frames of movie. Measured on 17 June 2015 at 7:53 (local time). Sun position $\theta=70^{\circ}, \phi=2^{\circ}$ (NOAA Solar Calculator). Wind speed $6 \mathrm{~m} / \mathrm{s}$ (wind direction from $280^{\circ}$ ).

The highest degree of linear polarization occurred close to the point of direct reflection of the sun. On the other hand directions close to nadir are almost free of polarization. But quite high values of DoLP $(>0.15)$ were derived from a ring formed by all observed azimuth directions for zenith angles between 60 and 90 degrees. The decreasing polarization with directions nearer to the nadir is consistent with simple Monte Carlo model presented by Kattawar et al. [4]. However, the exact comparison of presented results with Monte Carlo modelling would require additional input data containing, inter alia, IOPs and Mueller matrices of both atmosphere and seawater.

To see how the existence of high DoLP depended on solar glint with rough sea surface, we show four graphs of the average total intensity I (normalized to 1 for the highest value), the standard deviation of $I$, DoLP and its standard deviation (see Figure 8). These four views were calculated from a one movie of 1000 frames. The movie was recorded in the morning when the sun was only 20 degrees over a horizon.

The highest average intensity I occurred close to the horizon. It was due to the large distance to the places with flickering from the reflected sun glint. Closer to the position of the sun reflection, the area of strong sun blinks is visible as the region of high standard deviation of total intensity std(I). Although the area of the highest value of DoLP partially overlapped with the region of strong sun blinks, values of DoLP of 0.15 to 0.3 were observed in a large part of the view, forming a ring. Moreover standard deviations of DoLP had very low values in almost the entire area. The value of std(DoLP) are higher than 0.1 in place of the large variability caused by sun glints, and are relatively low in the rest of the ring. Hence the polarization of light was not derived from direct sun reflection, at least not in most of the visible area. It also was not controlled by the reflection of blue sky from sea surface, because the DoLP pattern of sky has totally different shape (see Figure 6 with its mini- mum close to sun and maximum 90 degrees off). It is therefore polarization coming from below the sea surface, and its origin is light refraction on the water surface and scattering by particulate (suspended) matter in the sea.

\section{CONCLUSIONS}

As a result of comparison of the experimentally derived rotation matrix with its theoretical form we conclude that maximum errors of measured elements of Stokes vector occurred on the border of the view, ie for light incoming from the horizon. These errors rapidly decreased with distance from the edge of view. Comparison of the measured polarization pattern of sky with its known form indicates that maximum errors of individual elements are compensated when we calculate the value of DoLP.

Measurements of DoLP of upwelling radiance over seawater indicate that the highest values of are observed in vicinity of sun reflection point and partially overlaps with a region of sun blinks. Directions close to the nadir have vary low polarization signal, but directions far from the nadir create a ring, in which the polarization of the radiance is relatively strong, and its source are not sun or sky reflections from the water surface. It is therefore the region, in which the polarization signal comes from the water column. That signal probably depends on the composition of sea water constituents. In the near future we are going to recognize the extent to which the signal is useful for remote sensing.

\section{ACKNOWLEDGMENTS}

Research presented in this paper was supported by a grant No. UMO-2012/07/D/ST10/02865, funded by National Science Centre (NCN) of Poland. Additionally, this paper was partially supported by the Academic Computer Centre in Gdańsk and by Statue Task I.3.2 of Institute of Oceanology PAS in Sopot, Poland.

\section{References}

[1] D. Deirmendjian, "Scattering and Polarization Properties of Water Clouds and Hazes in the Visible and Infrared," Appl. Optics 3, 187-196 (1964).

[2] G. W. Kattawar, and G. N. Plass, "Radiance and Polarization of Multiple Scattered Light from Haze and Clouds," Appl. Optics 7, 1519-1527 (1968).

[3] G. W. Kattawar, and G. N. Plass, "Degree and Direction of Polarization of Multiple Scattered Light. 1: Homogeneous Cloud Layers," Appl. Optics 11, 2851-2865 (1972).

[4] G. W. Kattawar, G. N. Plass, and J. A. Guinn Jr., "Monte Carlo calculations of the polarization of radiation in the Earth's atmosphereocean system," J. Phys. Oceanogr. 3, 353-372 (1973).

[5] G. W. Kattawar, and C. N. Adams, "Stokes Vector Calculations of the Submarine Light Field in an Atmosphere- Ocean with Scattering According to a Rayleigh Phase Matrix: Effect of Interface Refractive Index on Radiance and Polarization," Limnol. Oceanogr. 34, 1453-1472 (1989). 
[6] M. Chami, R. Santer, and E. Dilligeard, "Radiative Transfer Model for the Computation of Radiance and Polarization in an OceanAtmosphere System: Polarization Properties of Suspended Matter for Remote Sensing," Appl. Optics 40, 2398-2416 (2001).

[7] M. Chami, and D. McKee, "Determination of biogeochemical properties of marine particles using above water measurements of the degree of polarization at the Brewster angle," Opt. Express 15, 9494-9509 (2007).

[8] A. Gilerson, J. Zhou, M. 00, J. Chowdhary, B. M. Gross, F. Moshary, and S. Ahmed, "Retrieval of chlorophyll fluorescence from reflectance spectra through polarization discrimination: modeling and experiments," Appl. Optics 45, 5568-5581 (2006).

[9] M. Chami, "Importance of the polarization in the retrieval of oceanic constituents from the remote sensing reflectance," J. Ceophys. Res. 112, C05026 (2007).

[10] A. Tonizzo, A. Gilerson, T. Harmel, A. Ibrahim, J. Chowdhary, B. Gross, F. Moshary, et al. "Estimating particle composition and size distribution from polarized water-leaving radiance," Appl. 0ptics 50, 5047-5058 (2011).

[11] J. Piskozub, and W. Freda, "Signal of single scattering albedo in water leaving polarization," J. Eur. Opt. Soc.-Rapid 8, 13055, (2013).

[12] X. Q. He, D. L. Pan, Y. Bai, D. F. Wang, and Z. Z. Hao, "A new simple concept for ocean colour remote sensing using parallel polarisation radiance," Sci. Rep. 4, 3748 (2014).

[13] R. M. A. Azzam, "Photopolarimetric measurement of the Mueller matrix by Fourier analysis of a single detected signal," Opt. Letters 2, 148-150 (1978).

[14] K. J. Voss, and E. S. Fry, "Measurement of the Mueller matrix for ocean water," Appl. Optics 23, 4427-4439 (1984).
[15] A. Hielscher, A. Eick, J. Mourant, D. Shen, J. Freyer, and I. Bigio, "Diffuse backscattering Mueller matrices of highly scattering media," Opt. Express 1, 441-453 (1997).

[16] J. S. Tyo, D.L. Goldstein, D.B. Chenault, and J.A. Shaw, "Review of passive imaging polarimetry for remote sensing applications," Appl. Optics 45, 5453-5469 (2006).

[17] K. J. Voss, N. Souaidia, "POLRADS: polarization radiance distribution measurement system," Opt. Express 18, 19672-19680 (2010).

[18] P. Bhandari, "The Design of a Polarimeter and its Use for the Study of the Variation of Downwelling Polarized Radiance Distribution with Depth in the Ocean," Open Access Dissertations. 605 (2011).

[19] A. L. Iler, P. D. Hamilton, and A. Locke, "Use of Polarimetric Imaging for Discrimination of Oil from Water," in Fourier Transform Spectroscopy and Hyperspectral Imaging and Sounding of the Environment," HW2B.4, OSA Technical Digest (online) (2015).

[20] N. J. Brock, C. Crandall, and J. E. Millerd, "Snap-shot Imaging Polarimeter: Performance and Applications," P. Soc Photo-0pt. Ins. 9099, 909903 (2014).

[21] www.windfinder.com

[22] NOAA Solar Calculator www.esrl.noaa.gov.

[23] N. Pust, A. Dahlberg, M. Thomas, and J. Shaw, "Comparison of fullsky polarization and radiance observations to radiative transfer simulations which employ AERONET products," Opt. Express 19, 18602-18613 (2011).

[24] N. Pust, and J. Shaw, "All-sky polarization imaging," P. Soc PhotoOpt. Ins. 6682, 668204 (2007). 